\title{
Correction to: Is There a Relation Between Ecological Practices and Spirituality? The Case of Benedictine Monasteries
}

Bernhard Freyer ${ }^{1}$ (D) Valentina Aversano-Dearborn ${ }^{2} \cdot$ Georg Winkler $^{3}$. Sina Leipold ${ }^{4} \cdot$ Harald Haidl $^{2} \cdot$ Karl Werner Brand $^{5} \cdot$ Michael Rosenberger $^{3}$. Thomas Wallnig ${ }^{6}$

Published online: 11 April 2019

(C) The Author(s) 2019

\section{Correction to: Journal of Agricultural and Environmental Ethics (2018) 31:559-582 https://doi.org/10.1007/s10806-018-9745-4}

In the original publication of this article, the equally contributed article note was missed.

The missed article note is given in this Correction.

Bernhard Freyer and Valentina Aversano-Dearborn have contributed equally to this publication.

The original article can be found online at https://doi.org/10.1007/s10806-018-9745-4.

\section{Bernhard Freyer}

Bernhard.Freyer@boku.ac.at

Extended author information available on the last page of the article 
Publisher's Note Springer Nature remains neutral with regard to jurisdictional claims in published maps and institutional affiliations.

\section{Affiliations}

Bernhard Freyer $^{1}$ (D) . Valentina Aversano-Dearborn ${ }^{2} \cdot$ Georg Winkler $^{3}$. Sina Leipold ${ }^{4} \cdot$ Harald HaidI $^{2} \cdot$ Karl Werner Brand $^{5} \cdot$ Michael Rosenberger $^{3}$. Thomas Wallnig ${ }^{6}$

Valentina Aversano-Dearborn

Valentina.Aversano@boku.ac.at

Georg Winkler

g.winkler@ku-linz.at

Sina Leipold

sina.leipold@ifp.uni-freiburg.de

Harald Haidl

harald.haidl@aon.at

Karl Werner Brand

post@src-brand.de

Michael Rosenberger

m.rosenberger@ku-linz.at

Thomas Wallnig

thomas.wallnig@univie.ac.at

1 University of Natural Resources and Life Sciences, BOKU, Gregor-Mendelstr. 33, 1180 Vienna, Austria

2 BOKU, Gregor-Mendelstr. 33, 1180 Vienna, Austria

3 Katholische Privat-Universität Linz, Bethlehemstraße 20, 4020 Linz, Austria

4 Albert-Ludwigs-Universität Freiburg, Tennenbacher Str. 4, 79106 Freiburg, Germany

5 Sustainability Research Consulting (SRC), Haldenbergerstr. 55, 80997 Munich, Germany

6 Universität Wien, Maria-Theresien-Straße 9, 1090 Vienna, Austria 